\title{
Editorial
}

\section{Storm-Scale Radar Data Assimilation and High Resolution NWP}

\author{
Jidong Gao, ${ }^{1}$ David J. Stensrud, ${ }^{1}$ Louis Wicker, ${ }^{1}$ Ming Xue, ${ }^{2}$ and Kun Zhao ${ }^{3}$ \\ ${ }^{1}$ NOAA/National Severe Storm Laboratory, 120 David L. Boren Boulevard, Norman, OK 73072, USA \\ ${ }^{2}$ Center for Analysis and Prediction of Storms and School of Meteorology, University of Oklahoma, 120 David L. Boren Boulevard, \\ Norman, OK 73072, USA \\ ${ }^{3}$ Key Laboratory of Mesoscale Severe Weather/MOE, School of Atmospheric Sciences, Nanjing University, 22 Hankou Road, \\ Nanjing 210093, China \\ Correspondence should be addressed to Jidong Gao; jidong.gao@noaa.gov
}

Received 1 December 2013; Accepted 1 December 2013; Published 30 January 2014

Copyright (C) 2014 Jidong Gao et al. This is an open access article distributed under the Creative Commons Attribution License, which permits unrestricted use, distribution, and reproduction in any medium, provided the original work is properly cited.

With the steady increase in computing power, operational centers throughout the world are planning to run their numerical models with resolutions that begin to resolve the convective scale. This situation opens the opportunity and increases the demand for using radar data in convective-scale data assimilation in order to insert convective structures into model initial conditions. The potential for successfully assimilating radar data into convective-scale numerical weather prediction (NWP) models is predicated on the solution of several key challenges, including data quality control, proper estimation of the background error statistics, and, most notably, the estimation of atmospheric state variables that are not directly observed by radar. This special issue of 12 papers focuses on progresses in some of these important areas.

Radar data quality control is critical for radar data applications, including the use of radar data for severe weather warning, assimilation of radar data into stormscale NWP models, and diagnostic research on thunderstorm mechanisms and structures. Velocity dealiasing is an especially important and yet often very difficult part of the radar data quality control problem. In this special issue, Q. Xu et al. report on a Doppler velocity dealiasing technique based on alias-robust Velocity-Azimuth-Display (VAD) and variational analyses at the NOAA/National Severe Storms Laboratory (NSSL). This technique has been tested extensively with observations from the operational WSR88D radars under various weather conditions and data from a phased array radar (PAR) operated by NSSL. In another study related to radar data quality control, an automated technique is developed by Y. Jiang et al. to identify and remove contaminated velocities by birds, especially migrating birds in addition to those contaminated by ground clutter.

The effective assimilation of radar data into a NWP model requires advanced data assimilation (DA) techniques, such as variational and ensemble Kalman filter (EnKF) methods. A three-dimensional variational (3DVAR) system, which includes a mass continuity equation and the Advanced Regional Prediction System (ARPS) model equations as weak constraints, has been developed over the years at the Center for Analysis and Prediction of Storms (CAPS). The 3DVAR system was designed with special considerations for assimilating radar data into a convective-scale nonhydrostatic model, the ARPS, and has been used to provide initial conditions for numerous real time convective-scale NWP and research studies, including several papers in this special issue (G. Ge et al.; T. Hou et al.; E. Natenberg et al.; N. Yussouf et al.; M. Xue et al.). A major advantage of a 3DVAR system is its computational efficiency and the ease by which equation constraints can be included. However, one of the major shortcomings of a 3DVAR system is that it usually employs background error covariances that are not flowdependent, limiting their ability to "retrieved" unobserved state variables.

Compared to 3DVAR, the more advanced 4DVAR technique incorporates a full prediction model into the assimilation system and implicitly includes the effects of flowdependent error covariances through the use of both the forward prediction and backward adjoint models. In J. Sun 
and $\mathrm{H}$. Wang, a variational storm-scale data assimilation system for the Advanced Research Weather Research and Forecasting (WRF-ARW) model, including both 3DVAR and 4DVAR components, is described. The paper provides a review of the basics of the system and various studies that have been conducted to evaluate and improve its performance. Future activities that are required to further improve the system and to make it operational are also discussed. In another 4DVAR related work, X. Qiu et al. describe an application of the so-called simple adjoint method for retrieving three-dimensional winds from PAR data. They demonstrate that the method is computationally efficient and can be used for real-time applications with PAR rapid scans.

The EnKF is another advanced data assimilation method that shares many of the advantages of 4 DVAR. For convective storms, some encouraging results are reported using EnKF data assimilation in this special issue. The impact of model microphysics on storm-scale DA is a very important topic. In D. T. Dawson et al., the impact of increasing the number of predicted moments in a multimoment bulk microphysics scheme is investigated using EnKF analyses. They find that a triple-moment version of a microphysics scheme produces the best results in comparison to the single- and doublemoment versions. In another study, C. K. Potvin and L. J. Wicker apply procedures that have traditionally been used to retrieve the pressure and buoyancy fields from dual-Doppler wind analyses to rebalance the pressure fields produced by EnKF. The method is shown to provide an effective and lowcost way to remove pressure oscillations in the storm-scale EnKF analyses.

As pointed out earlier, 3DVAR is computationally efficient and can easily incorporate equation constraints that can be very beneficial. However, its use of static background error covariances limits its ability to retrieve unobserved variables. The EnKF directly uses flow-dependent error covariances derived from forecast ensemble but has a rank-deficiency problem due to the typically small ensemble size. To blend the advanced features of both variational and EnKF methods and to overcome their respective shortcomings, hybrid ensemblevariational (EnVar) algorithms had been proposed. Such an approach has been shown to be effective by a number of studies for large-scale data assimilation, but studies have been very limited for the storm scale. J. Gao et al. demonstrate the potential usefulness of a hybrid En3DVar method for convective-scale data assimilation. The algorithm uses the extended control variable approach to combine the static and ensemble-derived flow-dependent forecast error covariances, and the ensemble perturbations are provided by an EnKF that is coupled with the hybrid En3DVar. The method is applied to the assimilation of simulated radar data for a supercell storm. The results indicate that the hybrid method provides the best results among the 3DVAR, EnKF, and hybrid methods for hydrometeor-related state variables in terms of root-mean squared errors. For other state variables, the performance of the hybrid is very close to that of EnKF but much better than that of 3DVAR. More studies are needed in this direction.

The purpose of storm-scale data assimilation is to provide initial conditions for high resolution explicit convective storm prediction and quantitative precipitation forecasting.
In this aspect, several studies of high impact severe weather events are included in this special issue. To explore the impact of model physics on the mesoscale environmental variability and its uncertainty, combined mesoscale convective scale ensemble DA and forecast experiments are conducted for the 8 May, 2003, Oklahoma City tornadic supercell storm by N. Yussouf et al. Two sets of 36-member WRF-ARW model mesoscale ensemble adjustment Kalman filter (EAKF) DA experiments with continuous cycling on a continental United States domain are conducted to provide background environmental conditions, using either fixed physics or multiple physics parameterization schemes across the ensemble members, respectively. Results show that the ensemble with background fields from the multiple physics mesoscale ensemble provides more realistic forecasts of significant tornado parameter, dryline structure, and near surface variables than the ensemble from fixed physics mesoscale background fields. This suggests that incorporating physics diversity across the ensemble can be important to successful probabilistic convective-scale forecast of supercell thunderstorms. With the same 8 May, 2003, Oklahoma tornadic case, E. Natenberg et al. find that the use of data from all available radars provides the best forecast in which a strong low-level mesocyclone develops and tracks in close proximity to the actual tornado damage path. T. Hou et al. examine the impact of 3DVAR on the prediction of two heavy rainfall events over southern China by using the storm-scale WRF-ARW model. Observations from Doppler radars, surface Automatic Weather Station (AWS) network, and radiosondes are used. The assimilation of all radar, surface, and radiosonde data together produces more positive impacts on the forecast skill than the assimilation of only one type of data for the two rainfall events.

$\mathrm{M}$. Xue et al. report on the results of real-time convectionresolving forecasts at $1 \mathrm{~km}$ horizontal spacing over the continental United States in spring 2009 and a test case from spring 2008, produced by CAPS at the University of Oklahoma as part of the NOAA Hazardous Weather Testbed (HWT) Spring Experiment. The forecasts started from initial conditions which included the assimilation of radial velocity and reflectivity data from all operational WSR-88D radars within a large model domain. The assimilation used the ARPS 3DVAR with a cloud analysis package. Significant positive impact of radar data assimilation is found to last for at least 12 hours. Clear advantages of running the model at the convection-resolving $1 \mathrm{~km}$ grid over a coarser $4 \mathrm{~km}$ convection permitting grid spacing are also demonstrated. Convection-allowing storm-scale data assimilation and forecasting, including ensemble forecasting that provides probabilistic forecast information and radar data that provides valuable information on convective storms and precipitation, clearly represent the future of operational NWP.

\section{Acknowledgments}

As the guest editors for this special issue, we appreciate the contributions of all of the authors, reviewers, and journal staff. We sincerely hope that the publication of this special 
issue will encourage and invite more advanced research on storm-scale radar data assimilation and high resolution NWP.

Jidong Gao

David J. Stensrud

Louis Wicker

Ming Xue

Kun Zhao 

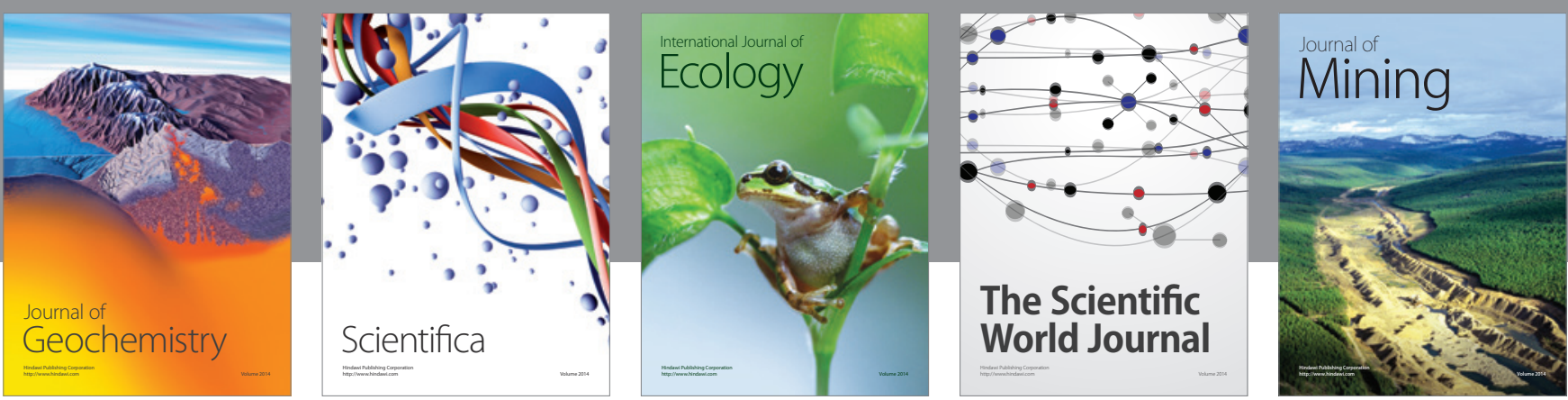

The Scientific World Journal
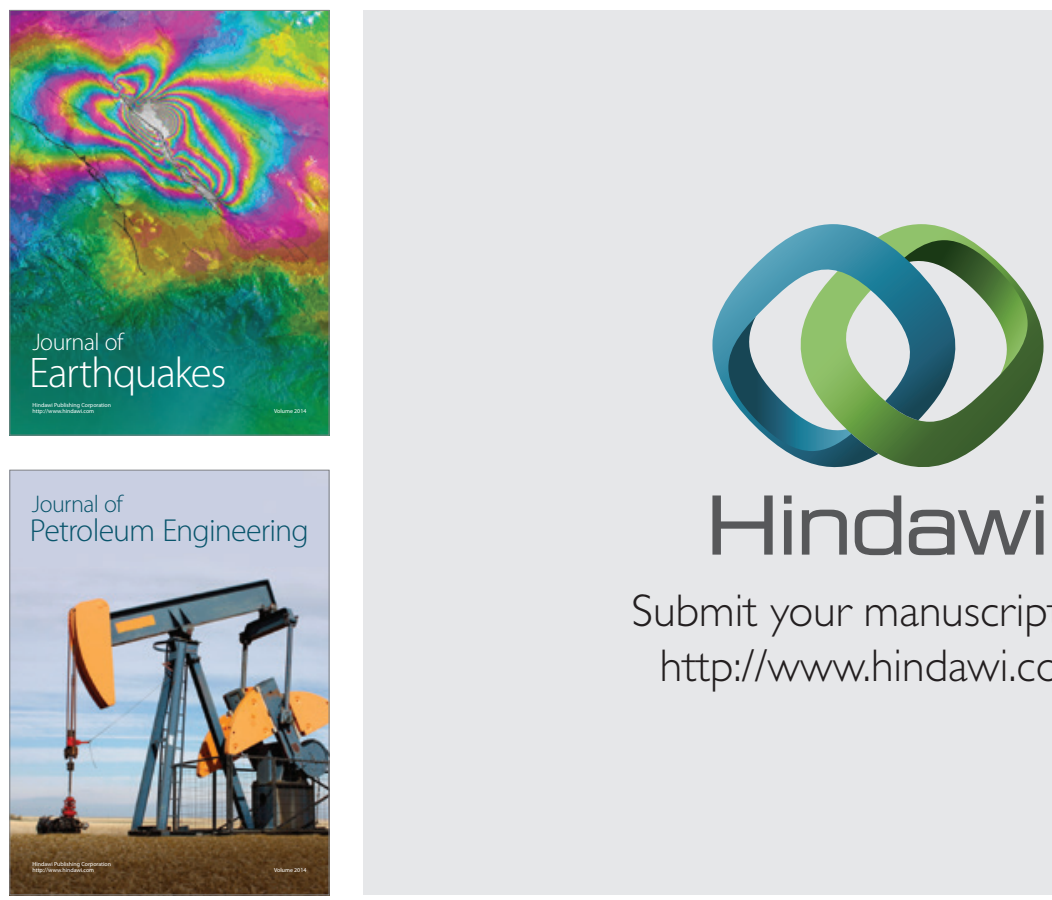

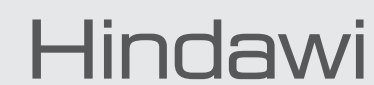

Submit your manuscripts at

http://www.hindawi.com
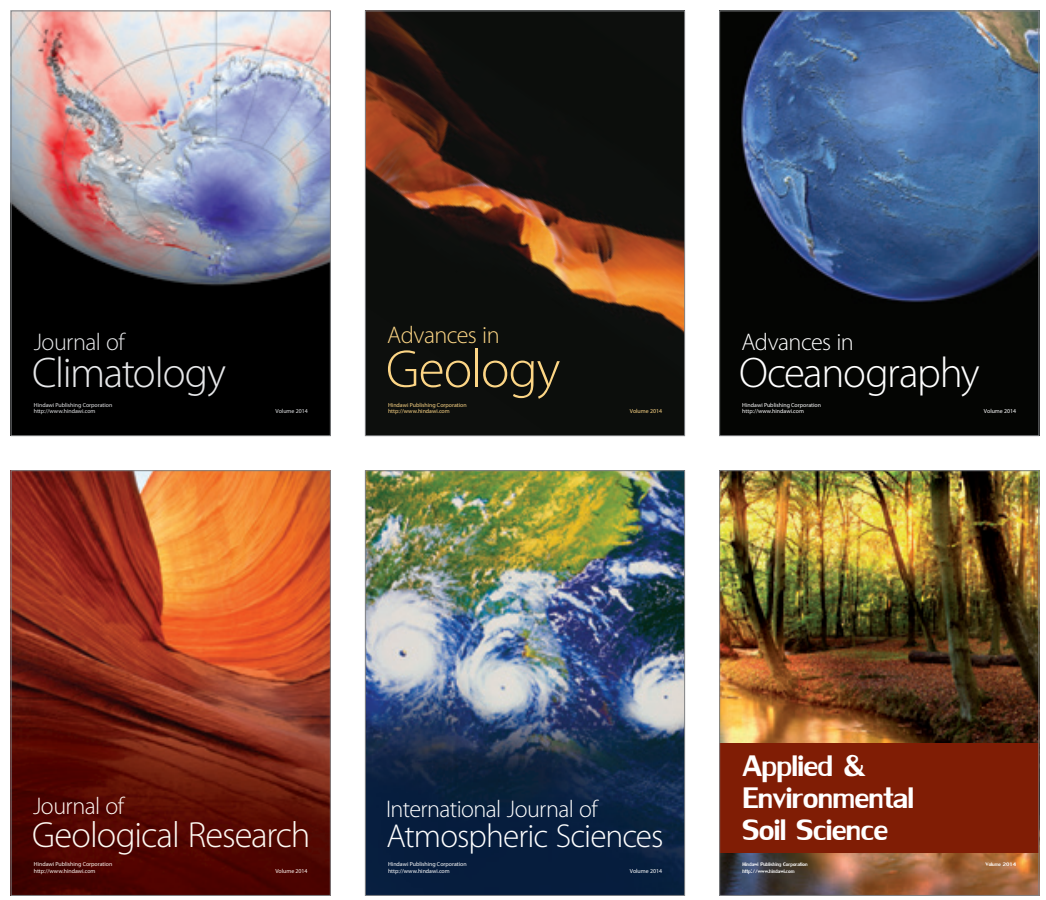
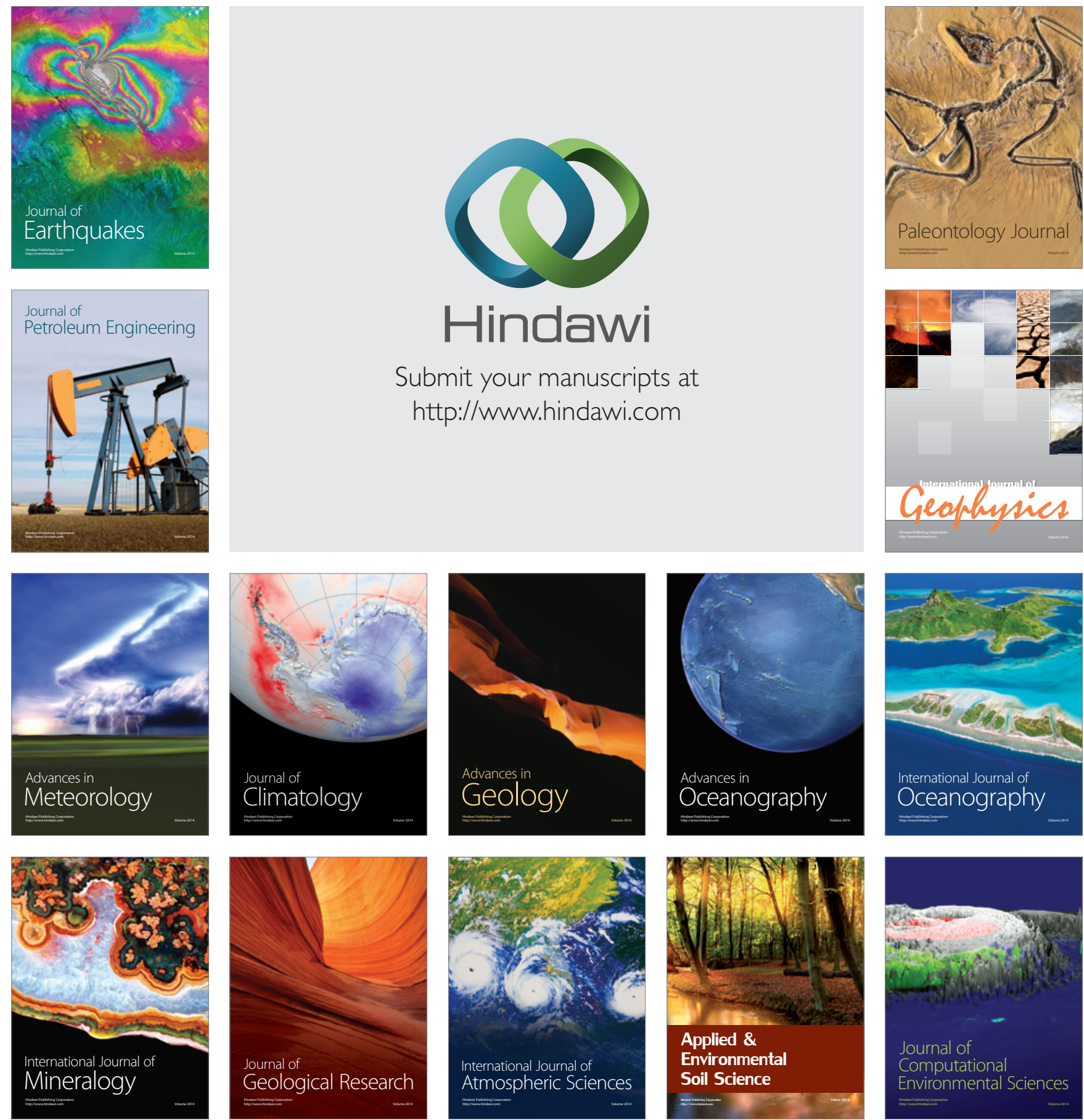